\title{
Research on the Layered Risk Control of "Blockchain + Digital Copyright" from the Perspective of Game Theory
}

\author{
Chen Hongying ${ }^{1, \mathrm{a}}$
}

\author{
${ }^{1}$ School of Law, Anhui University, Heifei, Anhui 230601 \\ aduoerxs@163.com
}

\begin{abstract}
The governance model of "blockchain + digital rights" has attracted wide attention due to its characteristics of decentralization and high efficiency. However, the problems of potential risk under the new technology governance model should also be paid attention to. Based on the technology model of blockchain, there are four risks of the four technical levels of the digital rights governance platform - operational effectiveness, supervision, privacy security, and smart contract. From the perspective of game theory, a tool to conduct hierarchical analysis, targeted risk control strategies for each level can be enclosed. It is hoped that the "blockchain + " model can be deeply integrated with digital copyright governance, and jointly promote the construction of the rule of law in China and the development of the academic research and practice of copyrights.
\end{abstract}

Keywords: blockchain, game theory, digital copyright governance, risk control

\section{INTRODUCTION}

Blockchain is a distributed ledger technology (DLT) with a system of decentralized management (usually selfmanagement), and it is the core technology for constructing Bitcoin data structure and transaction system[1]. With the birth and development of the Bitcoin financial system, blockchain has quickly entered the public eye. Due to the high popularity of the blockchain in the field of cryptocurrency, and its openness, transparency, traceability, and other characteristics, people have widely explored the application of blockchain in other fields, including the field of digital copyright management. At the beginning of 2020, the People's Copyright Platform in China formally connected to the Beijing Internet Court's “Tianping Chain" - an electronic evidence platform. By the first quarter of 2020, there have been over 2 million press released "on-chain" in People's Copyright with the technological help of blockchain[2]. There are still many cases of digital copyright deposits, transactions, and rights protection in the global world. The extensive use of the integration of blockchain technology and digital copyright governance has played a revolutionary role in solving the problems in confirming rights, maintaining rights, and exercising rights in digital copyright governance. However, the risks associated with the "blockchain + digital copyright" governance model also need practitioners and the public to stay alert.

\section{RISKS OF "BLOCKCHAIN + DIGITAL COPYRIGHT"}

After excluding pure economic incentives such as the mining mechanism, the "blockchain + digital copyright" platform usually builds a complete blockchain technology structure from the bottom to the top of the five-layer technology mode, which contains four foundations Level and final application level. The following will briefly sort out the four most representative risks hidden in each of the four basic technology levels of the "blockchain + digital copyright" governance model.

\subsection{Data Layer: Risks to the Effectiveness of Platform Operation}

The data layer is the lowest and most basic data structure of the blockchain. As the name suggests, it relies on a large amount of data to ensure the effective operation of the layer, and it is also the cornerstone of 
building subsequent layers. At this stage, most of the researches and discussions on blockchain in the field of digital copyright in the social sciences area are based on a fairly ideal assumption: the target platform as a whole continues to operate and is effective all the time. However, whether the "blockchain + digital copyright" governance platform can operate effectively, the initial data layer is faced with a huge test from the scale of users and the volume of information, that is, the application of blockchain in digital copyright governance has a Network externality risk. Network externality is evident in the information age, which means that the utility a person obtains from a certain commodity depends on the scale of consumers of this commodity[4]. Blockchain is a platform built by information network technology. If there is no comprehensive user information, digital copyright description, detailed digital copyright transaction information, and other information in the platform, and only the technical shell supported by the code, it will not be able to maintain the area. The operation of the blockchain is also difficult to attract more users to "on the chain" in order to realize the true value of the blockchain platform.

\subsection{Network Layer: the Risk of Law Enforcement and Supervision}

The issue of law enforcement and supervision is a major difficulty faced by blockchain technology to help digital copyright governance. It mainly acts on the construction of the second level of the peer-to-peer network (P2P): the network layer.

Because the blockchain is built in the P2P network, the information is no longer concentrated in the hands of a few people, but instead relies on the numerous equal nodes in the blockchain network to maintain the daily operation of the blockchain platform, which is a reflection of decentralization. The characteristic of decentralization is the root of the advantages of transparency, anti-counterfeiting, and high efficiency of the blockchain platform, but "decentralization" is an impact on the original "centralized" copyright governance system: according to the original system, notarization departments such as registration, taxation, and justice have the right and obligation to supervise or review copyright registration, transactions, and protection. Blockchain is extremely complex and is still in the early stage of technological development. Blockchain platforms, which is a technological platform lacking a central supervision mechanism, have to face multiple stakeholder groups without a neutral party. Since the blockchain itself is difficult to control this multi-party game to achieve a balanced state, the anonymization system that was originally conducive to the safe and efficient management of digital copyright, the non-tamperable and irrevocable record, etc., may become the cradle for piracy due to the lack of effective legal means. As a new hotbed of infringement and illegal communication, the derailment of the "blockchain+" platform will become a new difficulty and pain point in digital copyright governance and will bring numerous obstacles to law enforcement and supervision.

\subsection{Consensus Layer: Privacy and Security Risks}

The model is built on the consensus level, where the governance of the blockchain community is put into operation, and the connections and interactions between nodes are getting closer. With the advent of the Internet era of big data, platform users' information security protection and privacy security have become the concerns of the public. And the interactions in the consensus layer such as the storage of copyright information on the chain, the entry of user personal data, the real-time acquisition of geographic location when on the chain, and the mandatory disclosure of copyright transaction details will make digital copyright governance fall into "privacy protection" and the conflict between "openness and transparency". Should we "sell" personal privacy in exchange for fairness, efficiency, and convenience? Faced with this question, the consensus layer has not yet given a clear answer.

\subsection{Contract Layer: Smart Contract Risk}

Smart contracts are the main body of the fourth layer and the starting point of the trust system of the blockchain. There is no consensus on the legal definition of smart contracts in various fields at home and abroad, but its core connotation is clear: it is defined in the form of digital code according to emergencies based on decentralized consensus, low cost, and algorithm execution commitment (contract). A smart contract is essentially a text of a computer program or source code that is being executed[5]. From this, the discussion of "smart contract is a contract" has become a hot topic in the legal circles in the early days of the rise of blockchain. In most cases, a smart contract is the presentation of the code in the blockchain with a common meaning. The biggest difference between it and the ordinary contract is the high auto-execution of the smart contract. And this feature excludes the possibility of a party exercising the right of modification or revocation in digital copyright transactions once the preset conditions are met, no matter what the subjective will of the party is or what the situation is in reality, even in the face of fraud, coercion, or apparent unfairness. In other cases, the smart contract will also automatically complete a series of transaction activities for the target digital copyright. There are many places where the features 
of smart contracts conflict with the general contract law, copyright law, and other legal norms. In practice, as a major hidden danger, it quietly impacts the existing legal norms and market order and urgently needs to be included in the scope of legal regulation.

\section{HIERARCHICAL ANALYSIS FROM THE PERSPECTIVE OF GAME THEORY}

Society is an interactive body between people, and game theory is a tool for analyzing interactive decision-making, and it has been widely used in economics, law, sociology, and other fields[6]. From the perspective of human interaction, the most basic problem in society is coordination and cooperation. And the coordination and competition of all players in society, the reception and processing of information, the arrangement of action plans, and the combination of payoff with strategic equilibrium as the basic elements of the game, it is precisely different game competitions that are intertwined with each other. Game theory's analysis paradigm of "propose hypotheses-construct models/matrices-analyze and solve-discuss conclusions" provides methods and paths for solving complex social problems. Game theory is used as an analysis tool to analyze, predict and improve with the help of game theory models. The result of the game is to promote the effective allocation of resources, the construction of a society under the rule of law, and human progress.

\subsection{Data Layer: Strategic Adjustment Across Critical States}

Economies of scale are the characteristic that the long-term average total cost decreases as the output increases[7]. As mentioned above, only when the data layer successfully circumvents the potential risks of network externalities and gradually expands the scale of platform users, can the scale economy effect be realized, to active the core value of digital copyright governance together with other layers, otherwise the incomplete information will be scattered. In the blockchain platform, it can only exist independently as each "block", and cannot form a "chain structure", which directly leads to the "blockchain" in name only. Based on the assumption of non-ideal technology operation and the strategic goal of the equilibrium state of demand equal to supply, at the beginning of the platform' s development, there are very few users and digital copyright-related information reserves on the chain, although users and the scale of information may be gradually expanded due to the low usage and transaction costs, this is only an unstable and slow growth; only when a critical state is reached, the scale of the platform will rapidly expand and flourish. It can be seen that how to actively cultivate the industry and reach a critical scale in the early development of the blockchain governance of digital copyright has become a key point in surmounting network externality risks.

\subsection{Network Layer: The Game between Central Regulator and Platform/Market}

There are many different governance models, but how to maximize the respect for platform technology innovation based on complying with laws and regulations? We need to know that centralization and decentralization are always relative concepts. Internationally, it is generally believed that in order not to inhibit innovation opportunities, "restrictive" factors, such as central supervision, should be technologically neutral, and specific technical solutions should not be forced or prohibited. However, risks are carried out through the "connection point" between the system and the supervision and law enforcement agencies[8]. Evaluation, control, and processing of the maintenance of "blockchain + digital copyright" governance can rely on the reputation of the platform itself (good operation mode under the background of decentralization), or it can rely on the constraints of administrative and legal forces, which involves the game between central supervision and platform (market). As shown in the figure below, if the strategic combination of the central regulator and the platform is (not responding, reacting), people will not have a sense of security and trust in the blockchain platform, and the platform will therefore be difficult to establish a good reputation; if the regulatory strategy is implemented (response), law enforcement activities will enable more resources to be invested in blockchain-based digital copyright governance, which can effectively enhance the reputation of the platform. At the same time, it can also disclose negative information on the platform to the market, and then the market mechanism can regulate itself; however, when the supervision exceeds a certain level, due to the broader discretion, it may lead to the emergence of monopoly, punishment will also become untrustworthy, control itself will also cause corruption, and at the same time, it will be accompanied by the consumption of technological innovation. And hit, thus forming the trajectory of the two curves (two strategies), and the Nash equilibrium point is exactly the goal we are pursuing. 


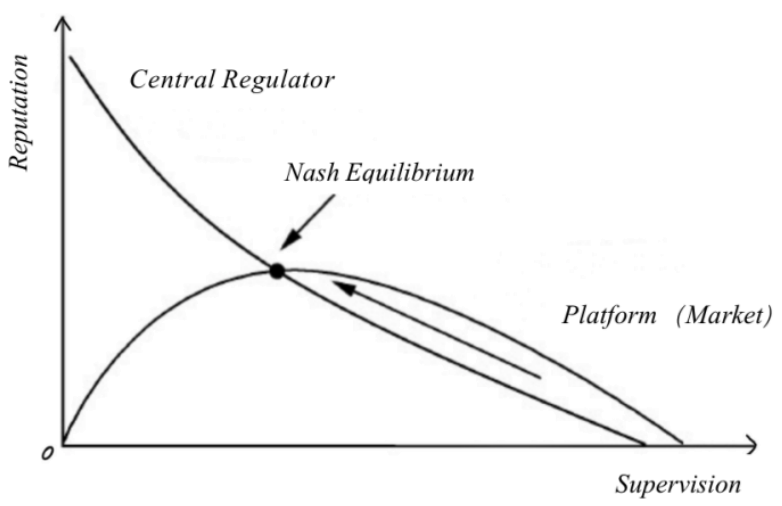

Figure 1 the Game between Central Regulator and Platform (Market)

\subsection{Consensus Layer: the Game between Information Disclosure and Privacy Protection}

Privacy and security represent the vital interests and value pursuit of the general public, and the use of blockchain for digital copyright governance has become the interests of copyright owners, parties to copyright transactions, judicial personnel and other related parties[4]. As shown in figure, the $\mathrm{X}$ axis for the chain information transparency level of strategy, D point for digital copyright information and users' personal information is public, $\mathrm{Y}$ axis for the chain of personal privacy and information protection policy, $\mathrm{C}$ point that the absolute protection of privacy, the user refused to chain or technical personal sharing of information dissemination way completely blocked. Users hope to obtain a complete trust system for evidence fixing, copyright registration, copyright trading, etc., and even the judicial organs, which can also accelerate the judicial efficiency through the docking with the "blockchain $+"$ platform. The fundamental purpose of "Blockchain + Digital Copyright" governance platform is to balance the interests of copyright owners and the interests of the public. The middle point A represents the balance of the two strategies.

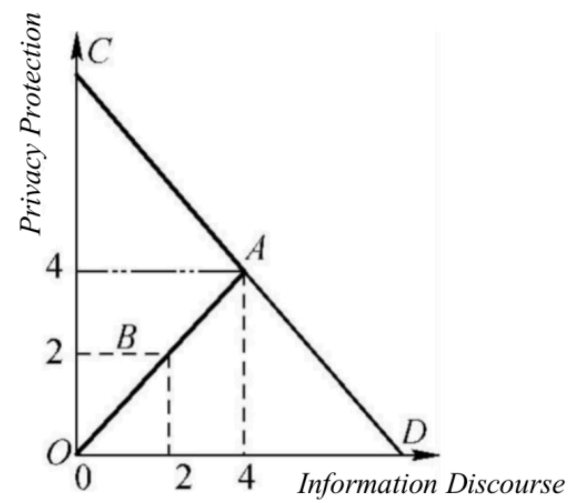

Figure 2 The Game between Information Discourse and Privacy Protection

\subsection{Contract Layer: the Game between Legal Norms and Technical Norms}

In the contract layer, how to bring smart contracts into the controllable range of the law involves the coordination of "law of law" and "law of technology". Should the law be endowed with technological capabilities beyond absolute rationality, or should the truth and rationality of technology be implemented to the end, so that the party whose interests have been harmed enters the field of tort law and then safeguards its rights and interests? In this context, if the state is allowed to intervene in the technical field, it will be inconsistent with the technical characteristics of blockchain decentralization and disintermediation, and will even cause a serious blow to the trust system painstakingly constructed by blockchain in the era of big data Internet; On the other hand, if the blockchain is allowed to open and automatically execute smart contracts, although it respects rational code logic and technical tolerance, it will increase the difficulty of rights protection for those who suffer from rights and interests and make it difficult to protect rights in the field of digital copyright transactions. The situation has worsened.

\section{RISK PREVENTION AND RISK CONTROL STRATEGY}

\subsection{Data Layer: Clear Technical Standards to Ensure Effectiveness of Platform Operation}

Pareto optimum has huge room for improvement. Pareto Optimality refers to a social state, compared with this state, there is no alternative state that can make at least one person's situation better while no other person's situation becomes worse[9]. How to reach a critical scale close to the Pareto optimum in the early stage of the development of digital copyright governance as soon as possible, while avoiding hidden dangers at the network layer? Satisfying the technical standards of legality, rationality, and adaptability will provide good guidance. At present, the state's 
development of blockchain technology standards is still at the basic stage and does not involve high-level areas such as trust and interaction. Clear technical standards have good authority and compatibility, attracting users and small-scale enterprises to quickly enter the platform and jointly participate in the construction of the platform, which help the "blockchain + digital copyright" governance model to move towards the Pareto optimal state as early as possible in the early stage of the life cycle.

\subsection{Network Layer: Resolves Regulatory Pain Points by Sandbox}

The Nash Equilibrium mentioned above is the optimal strategic combination of all participants. At this point, no matter whether it is the supervisory law enforcement officer who masters the control strategy or the market and platform itself that implements the reputation mechanism, neither party has the enthusiasm to change its choice. Although this point is the most ideal equilibrium state, due to practical factors such as high reform costs, it is difficult to directly establish a strategic equilibrium between regulators and platforms through factor evaluation. However, the regulatory sandbox creates a space for experimentation as close to equilibrium as possible. The regulatory sandbox is a cutting-edge achievement in foreign financial supervision. It is a "safe space" and "experimental field" in which companies can test innovative products, services, business models, and delivery mechanisms without immediately assuming all normal participation in related activities[10]. In recent years, the testing and layout of the regulatory sandbox in the Chinese financial sector has gradually extended to blockchain technology. The regulatory sandbox layout extends to the "blockchain+" digital copyright governance field, which will have a profound impact on exploring the optimal strategic combination of technology and the reputation of platforms and central supervision, test and manage regulatory risks, costs reduction, and the improvement of the quality and efficiency of digital copyright governance, etc.

\subsection{Consensus Layer: Build an Independent and Private "New Transaction"}

Many changes cannot meet the ideal Pareto criterion, so the Kaldor-Hicks criterion of efficiency (the criterion for maximizing the total amount) is introduced: the change can make the gains of the beneficiaries compensate for the losses of the losers. This standard is also a standard of collective rationality in social decisionmaking. Even if the compensation is not complete afterward, the copyright owners on the platform may not fully accept this method, but it can fix people' $s$ expectations of privacy risks and relief models, and guarantee equal opportunities for practice. Then even if the distribution is unequal afterward, the fairness of distribution can still be achieved from the perspective of expected utility, while realizing the maximization of the total social benefits. It can be indicated that the OA part can ensure the balance of information disclosure and privacy protection, showing a positively related cooperative relationship between the two.

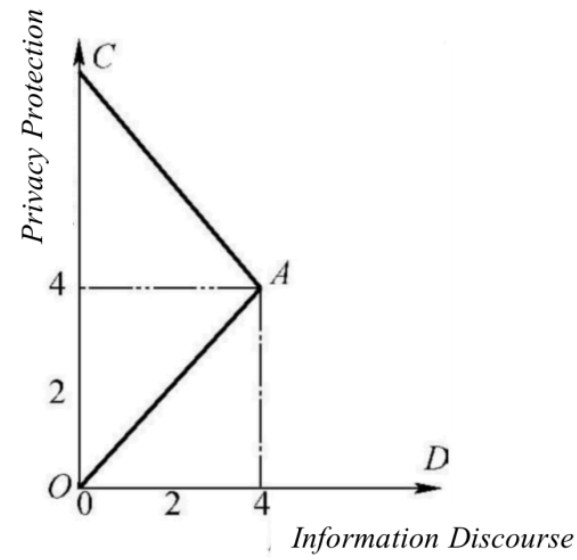

Figure 3 The Game between Information Discourse and Privacy Protection - Improvement

Although there are many difficulties in protecting privacy in the blockchain field, based on this consensus, existing teams have proposed a "Distributed KAnonymity Location Privacy Protection Scheme" in the field of anonymous location privacy protection. This technology encourages relevant users to $\log$ on to the chain to save real information and participate in the district. The construction of the blockchain anonymity zone separates the process of obtaining users' personal information from the activities on the chain to form a separate new "transaction", and save the transaction information to the public chain. As evidence storage, it also has the function of blocking the execution and punishment of users who leak privacy[11]. The specific technical means and code operation mode are not stated here, but this method primarily respects the "blockchain+" platform that brings our society an efficient information disclosure model that satisfies the breakthrough direction (OA) of Kaldor-Hicks improvement. Besides, the use of technical means to turn personal privacy acquisition behaviors that require special protection into a separate "transaction", forming an efficient and complete platform relief model. To make up for the possible losses caused by the information disclosure model, it has become one of the effective ways to achieve coordination among all stakeholders.

\subsection{Contract Layer: Targeted Support for Technical Improvement}

Based on the previous analysis, a game model of specific legal and technical specifications to obtain risk control strategies at the contract level can be built: 
Table 1 Important payment name and specific payment situation.

\begin{tabular}{|c|c|}
\hline Important payment & Specific payment \\
\hline the Cost of Legal Control & $C_{1}$ \\
\hline the Cost of Technical Control & $C_{2}$ \\
\hline Technological innovation initiative & $\mathrm{a}$ \\
\hline Rights protection (legal) risk assumption & $\beta_{7}$ \\
\hline Rights protection (technical) risk taking & $\beta_{2}$ \\
\hline Technical control policy assistance & $U$ \\
\hline Legal punishment (fine) & $P$ \\
\hline
\end{tabular}

Table 2 The strategic income matrix of legal norms and technical norms.

\begin{tabular}{|c|c|c|}
\hline Legal Technical & Regulation & Not regulation \\
\hline Regulation & $\begin{array}{c}\left(-C_{1}-\beta_{1}+P,-C_{2}+a+U-\beta_{2}\right. \\
+P)\end{array}$ & $\left(-C_{1}-\beta_{1}+P, \alpha+\beta_{2}-P\right)$ \\
\hline Not regulation & $\left(\beta_{1},-C_{2}+a+U-\beta_{2}\right)$ & $\left(\beta_{1}, a+\beta_{2}\right)$ \\
\hline
\end{tabular}

Due to space limitations, the classified discussion and payment comparison of each strategy combination are omitted here. Integrate the previous analysis of the strategic income matrix of the two norms of law and technology, and exclude the strategic combination of regulatory failure, unsustainable, and inconsistent with domestic practice. When policy assistance $U>$ technology self-regulation cost $\mathrm{C} 2$, (no regulation, regulation) The combination of strategies is most likely to meet the sustainable development needs of digital copyright governance and blockchain technology. This strategy not only satisfies the needs of copyright protection, but also completes the constraints of the technology itself before entering the field of legal regulation, and achieves the best-balanced result of the game between the two parties. From this analysis, it can be seen that if blockchain technology can be self-optimized within the existing legal and institutional system to increase guidance and support, and form a strategic combination that meets the requirements of sustainable development, it will effectively alleviate the friction and conflict between technology and law. Reach a consensus on social development.

\section{CONCLUSION AND OUTLOOK}

As a part of intellectual property rights, copyright has an inseparable natural connection with cultural innovation. Optimizing modern digital copyright governance models is more in line with the needs of social and economic development of modern digital transformation. At the same time, the domestic blockchain research and application has entered an important period of opportunity and has become an important breakthrough for the independent innovation of the country's core technology. Seeing that the "blockchain + digital copyright" governance model reflects the inevitable development trend of the deep integration of technology and social governance, and the prospects are great, we also need to maintain a rational and dialectical vision, and carefully analyze the hidden risks of each basic technology level. Besides, we will carry out targeted prevention and control, coordinate the interests of multiple parties, and strive to achieve the optimal strategic balance, and finally move towards a better social state, empowering the development of my country's copyright industry, the construction of an innovative country, and the construction of a modern rule of law.

\section{REFERENCES}

[1] Cong, Lin William, and Zhiguo He. Blockchain disruption and smart contracts, The Review of Financial Studies 32.5 (2019), 1754-1797.

[2] Yang Yubo, Lu Qian. "People's copyright" records big data through blockchain service, making "taking doctrine" inevitable. http://it.people.com.cn/GB/n1/2020/0427/c100931690197.html, 2020-04-27.

[3] Shafagh, H., Burkhalter, L., Hithnawi, A., \& Duquennoy, Towards blockchain-based auditable storage and sharing of iot data. the 2017 on Cloud Computing Security Workshop (pp. 45-50).

[4] Hal R. fan Lian. Microeconomics: a modern 
perspective. Shanghai People's publishing house, 9th Edition, 2014, 481-482.

[5] Bodó B, Gervais D, Quintais J P, Blockchain and smart contracts: the missing link in copyright licensing? International Journal of Law and Information Technology, 2018, 26(4), 315-316.

[6] Zhang Weiying. game and society, Peking University Press, January 2013, 42-43.

[7] Man Kun. principles of Economics: 7th Edition. Microeconomics volume, Peking University Press, 2015, 291.

[8] Gürkaynak G, Yılmaz İ, Yeşilaltay B, et al. Intellectual property law and practice in the blockchain realm, Computer Law \& Security Review, 2018, 34(4): 847-862.

[9] Xie Shiyu. economic game theory, Shanghai Fudan University Press, 4th Edition, 2019, 85-87.

[10] Authority F C. Regulatory sandbox. Financial Conduct Authority, 2015, 19.

[11] Liu Hai, Li Xinghua, et al. Distributed k-anonymity location privacy protection scheme based on blockchain, Journal of computer science, No. 5, 2019, 942-960. 